\title{
COMPUTATION OF EIGENVALUES IN LINEAR ELASTICITY WITH LEAST-SQUARES FINITE ELEMENTS: DEALING WITH THE MIXED SYSTEM
}

\section{LINDA ALZABEN ${ }^{1}$, FLEURIANNE BERTRAND ${ }^{2}$ AND DANIELE BOFFI $^{3}$}

${ }^{1}$ King Abdullah University of Science and Technology

Thuwal 23955-6900, Kingdom of Saudi Arabia

linda.alzaben@kaust.edu.sa

${ }^{2}$ University of Twente

7500 AE Enschede, The Netherlands

f.bertrand@utwente.nl

${ }^{3}$ King Abdullah University of Science and Technology and University of Pavia

Thuwal 23955-6900, Kingdom of Saudi Arabia and Via Ferrata 1, I-27100 Pavia, Italy daniele.boffi@kaust.edu.sa

Key words: eigenvalue problems, linear elasticity, least-squares finite elements, mixed finite element method

Abstract. In this paper we discuss some aspects related to the practical implementation of a method that has been introduced recently for the approximation of the eigenvalues of the linear elasticity problem. The scheme, based on a least-squares finite element formulation, gives rise to a non-symmetric discrete formulation that may have complex eigenvalues. Moreover the algebraic eigenvalue problem to be solved is singular, so that the theoretical estimates about the convergence of the scheme should be carefully interpreted.

\section{INTRODUCTION}

This paper continues the research started in [3] about a least-squares finite element formulation for the approximation of the eigenvalue problem associated with linear elasticity. The formulation was introduced in [1] for the source problem. It is a two field formulation where, using as unknowns the displacement vector and the stress tensor, the second order elasticity equation is decomposed into a first order system.

In this note we recall the main results of [3] and discuss more specifically the definition of convergence of the numerical scheme. Indeed, the algebraic system that is obtained after the finite element discretization has the following structure which resembles an eigenvalue problem in mixed form

$$
\left(\begin{array}{cc}
A & B^{\top} \\
B & C
\end{array}\right)\left(\begin{array}{c}
\hat{\boldsymbol{\sigma}} \\
\hat{\mathbf{u}}
\end{array}\right)=\omega\left(\begin{array}{cc}
0 & D \\
0 & 0
\end{array}\right)\left(\begin{array}{c}
\hat{\boldsymbol{\sigma}} \\
\hat{\mathbf{u}}
\end{array}\right)
$$


At least two issues are associated with the analysis of such a problem: first of all the matrix on the right hand side is clearly singular so that the definition itself of the solution should be carefully stated. Then, it is apparent that the system cannot be reduced to a symmetric form due to the fact that the matrix $D$ is different from $B^{\top}$. If the two matrices were related to each other, then a similar strategy as the one performed in [2] for the Laplace operator would lead to a symmetric problem.

A typical technique to deal with mixed problem consists in the introduction of appropriate Schur complements. In this case we present two possible Schur complement factorizations and we show how they can be used to solve the system. Analogies and differences are described.

Finally, we recall the definition of convergence and we discuss how to apply this to the case when complex eigenvalues are present in the discrete system.

\section{PROBLEM SETTING}

Let $\Omega$ be an open, bounded, and connected subset of $\mathbb{R}^{2}$ with its boundary $\partial \Omega$ being Lipschitz continuous. The boundary of the domain $\Omega$ is divided into two sets, $\Gamma_{D}$ and $\Gamma_{N}$. By considering the first-order system for stress/displacement of linear elasticity with its boundary conditions in [1], we have the following: find a displacement vector field $\mathbf{u}$ and a symmetric $2 \times 2$ tensor $\underline{\sigma}$ such that

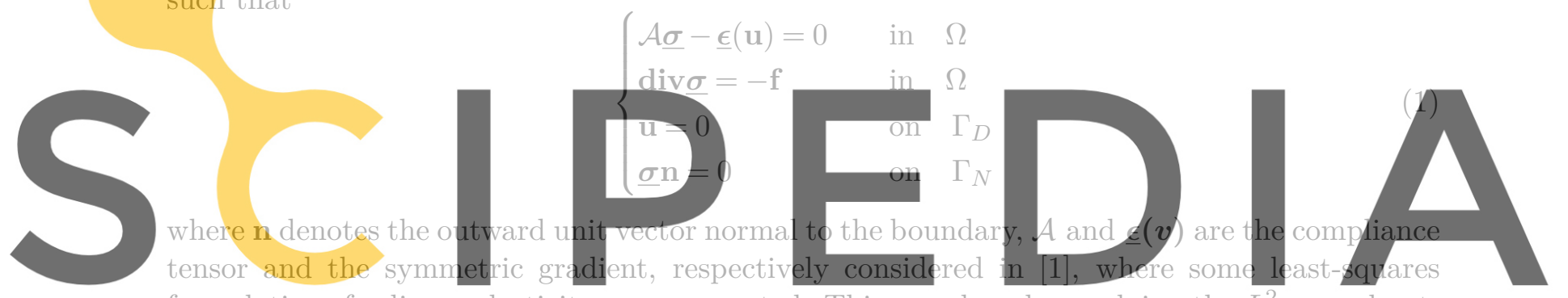

formulations for linear elasticity were presented. This was done by applying the $L^{2}$ norm least-

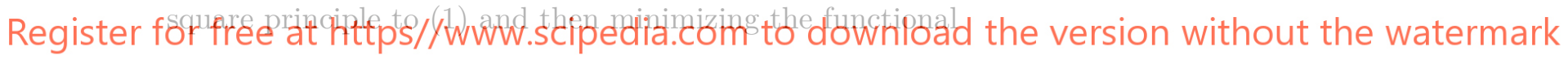

$$
\mathcal{F}(\underline{\tau}, \mathrm{v} ; \mathrm{f})=\|\mathcal{A} \underline{\underline{\tau}}-\underline{\epsilon}(\mathrm{v})\|_{0}^{2}+\|\operatorname{div} \underline{\tau}+\mathrm{f}\|_{0}^{2}
$$

in $\underline{\boldsymbol{X}}_{N} \times H_{0, D}^{1}(\Omega)^{2}$, with

$$
\underline{\boldsymbol{X}}=\left\{\begin{array}{lll}
\boldsymbol{H}(\boldsymbol{d i v} ; \Omega)^{2} & \text { if } & \Gamma_{N} \neq \emptyset \\
\left\{\underline{\boldsymbol{\tau}} \in \boldsymbol{H}(\operatorname{div} ; \Omega)^{2}: \int_{\Omega} \operatorname{tr}(\underline{\boldsymbol{\tau}}) \mathrm{d} \mathbf{x}=0\right\} & \text { if } & \Gamma_{N}=\emptyset
\end{array}\right.
$$

where $\underline{\boldsymbol{X}}_{N}$ is the subset of $\underline{\boldsymbol{X}}$ corresponding to the boundary condition $\underline{\boldsymbol{\tau}} \mathbf{n}=0$ on $\Gamma_{N}$.

\subsection{Eigenvalue problem for linear elasticity}

We are interested in studying the properties of the spectrum of the operator arising from the least-squares approximation of (1). This is done by replacing the source term $\mathbf{f}$ in (1) with the expression $\omega \mathbf{u}$ and by writing the corresponding least-squares formulation. The problem reads: 
find $\omega \in \mathbb{R}$ such that for non vanishing $\mathbf{u}$ and for some $\underline{\boldsymbol{\sigma}}$ we have

$$
\left\{\begin{array}{lll}
\mathcal{A} \underline{\boldsymbol{\sigma}}-\underline{\epsilon}(\mathbf{u})=0 & \text { in } & \Omega \\
\operatorname{div} \underline{\boldsymbol{\sigma}}=-\omega \mathbf{u} & \text { in } & \Omega \\
\mathbf{u}=0 & \text { on } & \Gamma_{D} \\
\underline{\boldsymbol{\sigma}} \boldsymbol{n}=0 & \text { on } & \Gamma_{N}
\end{array}\right.
$$

Due to the symmetry of linear elasticity problems, we seek for eigenvalues which are real $(\omega \in \mathbb{R})$. This idea has been exploited originally in [2] in the case of the Laplace operator and extended to non symmetric problems associated with linear elasticity in [3]. This was done by studying the spectrum of the operators associated with the least-squares source formulation, which deduced the so called two-field formulation.

\subsection{Two-field formulation and the eigenvalue problem}

The two-field formulation of the source problem considered in [1] consists in minimizing the functional $\mathcal{F}(\underline{\boldsymbol{\tau}}, \mathbf{v} ; \mathbf{f})$. This gives rise to the variational formulation which reads: find $\underline{\sigma} \in \underline{\mathbf{X}}_{N}$ and $\mathbf{u} \in H_{0, D}^{1}(\Omega)^{2}$ such that

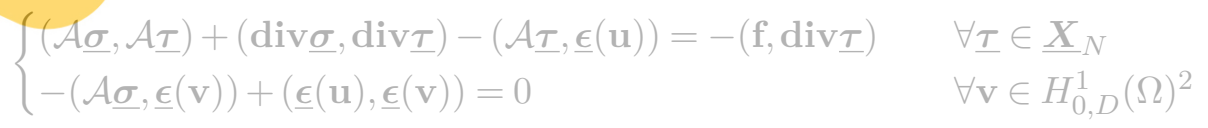

The eigenvalue variational formulation associated with the two-field is obtained by replacing

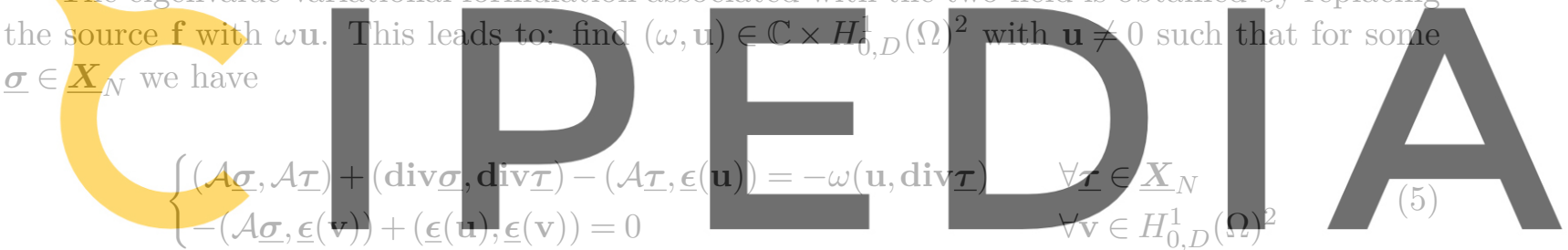

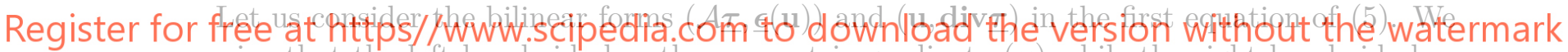
perceive that the left hand side has the symmetric gradient $\underline{\epsilon}(\mathbf{u})$ while the right hand side has $\operatorname{div} \tau$ in the bilinear forms. As a consequence, the problem is non-symmetric, hence, some of the computed eigenvalues may be in the complex plane even if we are approximating a symmetric problem. This is a natural consequence of the least-squares method.

\section{NUMERICAL APPROXIMATION}

The Galerkin discretization of eigenvalue problem introduced in (5) is as follows. Let $\Sigma_{h} \subset$ $\underline{\boldsymbol{X}}_{N}$ and $U_{h} \subset H_{0, D}^{1}(\Omega)^{2}$ be conforming finite element spaces. Then the discrete formulation reads: find the pair $\left(\omega_{h}, \mathbf{u}_{h}\right) \in \mathbb{C} \times U_{h}$ with $\mathbf{u}_{h} \neq 0$ such that for some $\underline{\boldsymbol{\sigma}}_{h} \in \Sigma_{h}$ we have

$$
\begin{cases}\left(\mathcal{A} \underline{\boldsymbol{\sigma}}_{h}, \mathcal{A} \underline{\boldsymbol{\tau}}\right)+\left(\operatorname{div} \underline{\boldsymbol{\sigma}}_{h}, \operatorname{div} \underline{\boldsymbol{\tau}}\right)-\left(\mathcal{A} \underline{\boldsymbol{\tau}}, \underline{\boldsymbol{\epsilon}}\left(\mathbf{u}_{h}\right)\right)=-\omega_{h}\left(\mathbf{u}_{h}, \operatorname{div} \underline{\boldsymbol{\tau}}\right) & \forall \underline{\boldsymbol{\tau}} \in \Sigma_{h} \\ -\left(\mathcal{A} \underline{\boldsymbol{\sigma}}_{h}, \underline{\boldsymbol{\epsilon}}(\mathbf{v})\right)+\left(\underline{\boldsymbol{\epsilon}}\left(\mathbf{u}_{h}\right), \underline{\boldsymbol{\epsilon}}(\mathbf{v})\right)=0 & \forall \mathbf{v} \in U_{h}\end{cases}
$$

The eigenvalue problem given in (6) has the typical structure of a mixed form given by

$$
\left(\begin{array}{cc}
A & B^{T} \\
B & C
\end{array}\right)\left(\begin{array}{c}
\hat{\boldsymbol{\sigma}} \\
\hat{\mathbf{u}}
\end{array}\right)=\omega\left(\begin{array}{cc}
0 & D \\
0 & 0
\end{array}\right)\left(\begin{array}{c}
\hat{\boldsymbol{\sigma}} \\
\hat{\mathbf{u}}
\end{array}\right)
$$


where the various operators correspond to the bilinear forms of our formulation as follows

$$
\left\{\begin{array}{l}
A \leftrightarrow\left(\mathcal{A} \underline{\boldsymbol{\sigma}}_{h}, \mathcal{A} \underline{\boldsymbol{\tau}}\right)+\left(\operatorname{div} \underline{\boldsymbol{\sigma}}_{h}, \operatorname{div} \underline{\boldsymbol{\tau}}\right) \\
B \leftrightarrow-\left(\mathcal{A} \underline{\boldsymbol{\sigma}}_{h}, \underline{\boldsymbol{\epsilon}}(\mathbf{v})\right) \\
C \leftrightarrow\left(\underline{\boldsymbol{\epsilon}}\left(\mathbf{u}_{h}\right), \underline{\boldsymbol{\epsilon}}(\mathbf{v})\right) \\
D \leftrightarrow-\left(\mathbf{u}_{h}, \operatorname{div} \underline{\boldsymbol{\tau}}\right)
\end{array}\right.
$$

As explained in Section 2.2, the operators $B^{T}$ and $D$ are different which leads to a non symmetric problem. The numerical approximation in (7) gives rise to an eigenvalue problem of the form

$$
\mathbb{A} x=\omega \mathbb{B} x
$$

The matrix $\mathbb{B}$ in our framework is clearly singular, however, $\mathbb{A}$ is not. For this reason, it may be appropriate to swap the roles of $\mathbb{A}, \mathbb{B}$ by considering the problem

$$
\mathbb{B} x=\eta \mathbb{A} x
$$

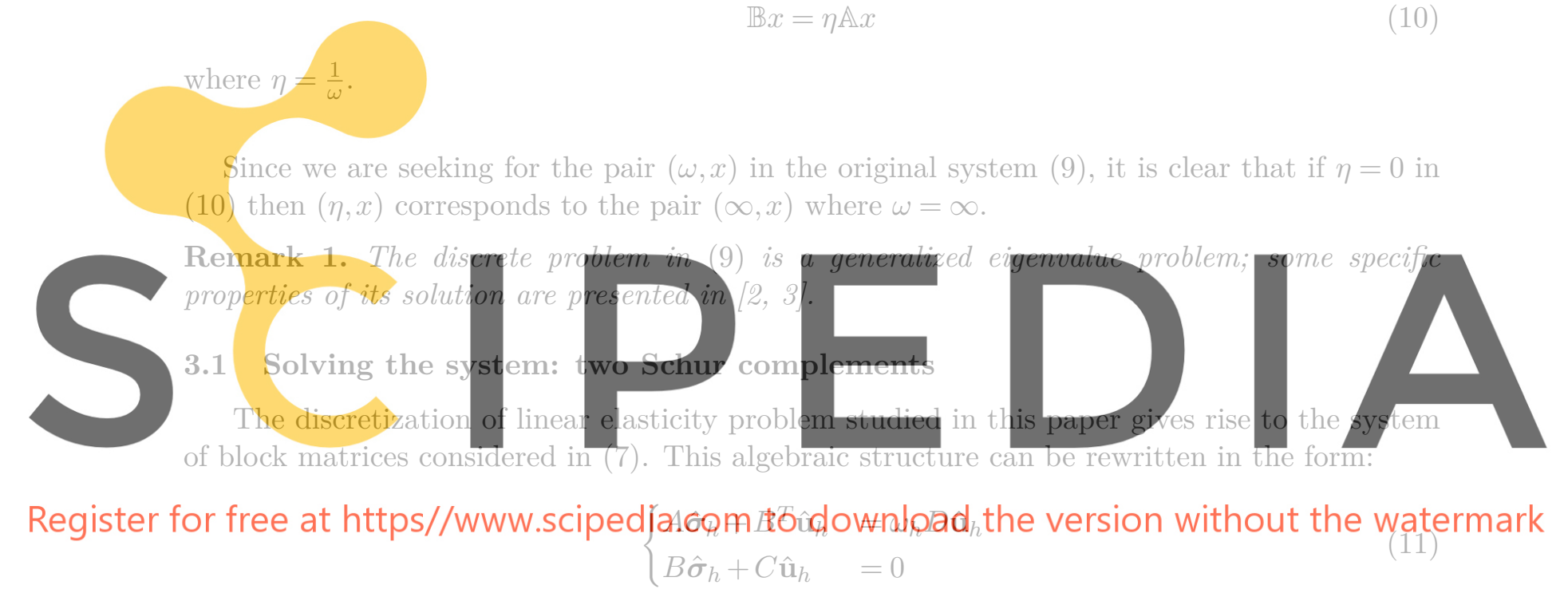

We are seeking for the discrete eigenvalues $\omega_{h}$ in the complex plane with a non-vanishing $\hat{\mathbf{u}}_{h}$. Moreover, as it has been explained at the end of the previous section, the solution of the algebraic eigenvalue problems associated with the variational formulation given in (6), will also include eigenvalues $\omega_{h}=\infty$. By Proposition 1 in [3], the multiplicity of such eigenvalues is equal to $\operatorname{dim}\left(\Sigma_{h}\right)+\operatorname{dim}(\operatorname{ker}(D))$ where ker is the kernel of the matrix. In addition, the number of finite eigenvalues (counted with their multiplicity) is equal to the rank of the matrix $D$.

The Schur complement technique is one of the strategies to deal with mixed problems. At this stage we approach the problem by finding two Schur complements associated with the system in (11), one related to the variable $\hat{\boldsymbol{\sigma}}_{h}$ and the other to $\hat{\mathbf{u}}_{h}$.

1. The first Schur complement considered, denoted by $S c h u r_{\sigma}$ and the eigenpair by $\left(\omega_{h}^{\sigma}, \hat{\boldsymbol{\sigma}}_{h}\right)$, is obtained after a straightforward calculation when solving (11), and is given by

$$
\left(A-B^{T} C^{-1} B\right) \hat{\boldsymbol{\sigma}}_{h}=-\omega_{h}^{\sigma} D C^{-1} B \hat{\boldsymbol{\sigma}}_{h}
$$


After solving (12), we can recover the other component of the solution by

$$
\hat{\mathbf{u}}_{h}=-C^{-1} B \hat{\boldsymbol{\sigma}}_{h}
$$

Note that by this approach, we first seek for eigenvalues associated with the $\hat{\boldsymbol{\sigma}}_{h}$ variable and then look for the pair of our interest. In other words, we first find the eigenpair $\left(\omega_{h}^{\sigma}, \hat{\boldsymbol{\sigma}}_{h}\right)$ and then $\left(\omega_{h}^{\sigma}, \hat{\mathbf{u}}_{h}\right)$ for $\hat{\mathbf{u}}_{h} \neq 0$.

Remark 2. This procedure might introduce some solutions which do not correspond to the ones we are interested in. Indeed, in common applications, the dimension of the space $\Sigma_{h}$ is larger than the dimension of $U_{h}$ so that more eigenvalues than the ones we are interested in may be computed by this Schur complement approach.

2. The second Schur complement is associated with the variable $\hat{\mathbf{u}}_{h}$ and the pair is denoted by $\left(\omega_{h}^{u}, \hat{\mathbf{u}}_{h}\right)$. By using (11) and with $A$ being invertible we have

$$
\hat{\sigma}_{h}=\left(\omega_{h} A^{-1} D-A^{-1} B^{T}\right) \hat{\mathbf{u}}_{h}
$$

and, after some manipulation, we arrive at the following algebraic system

$$
\left(B A^{-1} B^{T}-C\right) \hat{\mathbf{u}}_{h}=\omega_{h}^{u} B A^{-1} D \hat{\mathbf{u}}_{h}
$$

Since this system involves only the variable $\hat{\mathbf{u}}_{h}$, we refer to it as $\operatorname{Schur}_{u}$ complement approach.

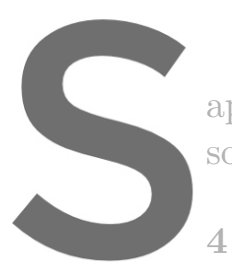

When comparing approach guarantees solve System (11) and 4. CONVERGENCH
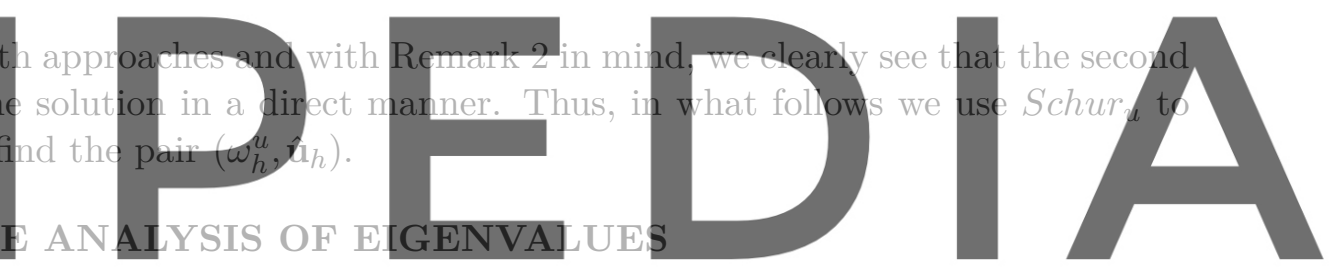

Looking at the convergence analysis, we will only discuss the uniform convergence of the

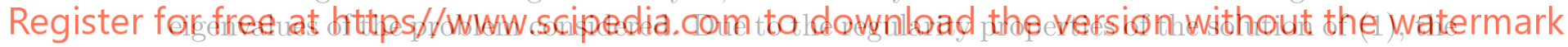
continuous eigenvalue problem in (3) is compact and therefore its eigenvalues form an increasing sequence

$$
0<\omega_{1} \leq \omega_{2} \leq \omega_{3} \leq \cdots \leq \omega_{i} \leq \cdots \quad \text { with } \quad \lim _{i \rightarrow \infty} \omega_{i}=\infty
$$

where the eigenvalues are real and positive and the eigenspaces are finite dimensional. As always, the same eigenvalue can be repeated several times according to its multiplicity so that we have one dimensional eigenspace for each eigenvalue (see $[4,5]$ for details).

Moving to the discrete problem (6), the discrete eigenvalues are complex and ordered according to their magnitude as follows

$$
0<\left|\omega_{1, h}^{u}\right| \leq\left|\omega_{2, h}^{u}\right| \leq\left|\omega_{3, h}^{u}\right| \leq \cdots
$$

Thus, convergence of eigenvalues means that if we take a number of eigenvalues, counted with their multiplicity, within a circle of radius $R>0$, for $h$ small enough there is exactly the same number of discrete eigenvalues approximating them. 
This follows from the general theory (see [4], Theorem 9.1) related to the approximation of eigenvalues of compact operators.

Theorem 4.1. For all compact sets $K$ in the complex plane, that is $K \subset \mathbb{C}$, that does not contain any eigenvalue of the continuous problem, there exits $h_{0}$ such that $\forall h<h_{0}$ no eigenvalue of the discrete problem belongs to $K$.

This notion of convergence can be made more precise as follows.

$\forall R>0$ let $\omega_{1}, \ldots, \omega_{N}$ be the $N$ eigenvalues, counted with their multiplicity, satisfying $\left|\omega_{i}\right|<$ $R$. Then, $\forall \epsilon>0, \exists h_{0}$ such that for $h<h_{0}$ exactly $N$ discrete eigenvalues, counted with their multiplicity, satisfy $\left|\omega_{i, h}^{u}\right|<R$. Moreover, the $N$ discrete eigenvalues can be sorted such that the following inequality is satisfied

$$
\left|\omega_{i}-\omega_{i, h}^{u}\right|<\epsilon \quad i=1, \ldots, N
$$

We have performed a preliminary set of numerical experiments that confirm the theory. Some tests are reported in [3] and more detailed experiments will be presented in a forthcoming paper. The numerical evidence shows that discrete eigenvalues can be present everywhere in the complex plane, including negative values, eigenvalues with negative real part, and, as expected, infinite eigenvalues. However, in all cases it is possible to find a circle centered at zero such that inside the circle only relevant eigenvalues are present. Moreover, as $h$ goes to zero, the radius of the circle can be taken larger and larger. This is in perfect agreement with the convergence theory

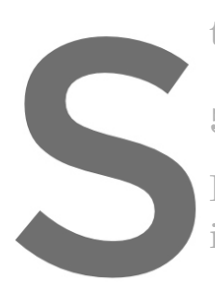
that has been described abore

5 CONCLUSIONS

In this paper we investig

in [3]. We succeed in fir

Schur $_{\sigma}$ and Schur
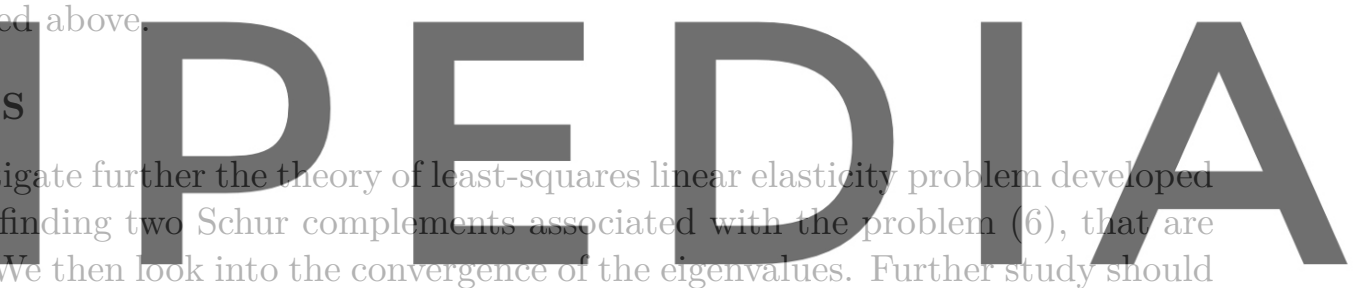

account for the convergence order of eigenvalues and eigenvectors applied to the above theory

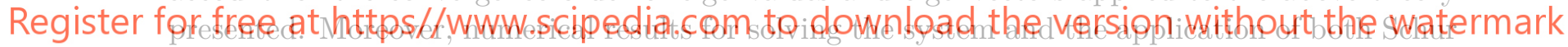

shall be analyzed. Another interesting proposition is to look into the mesh being considered

in such problems. Since the problem itself is non symmetric, a valid question would be what are the eigenvalues and eigenvectors if a symmetric/non symmetric mesh is applied to such a problem.

\section{REFERENCES}

[1] Cai, Z. and Starke, G., 2004. Least-squares methods for linear elasticity. SIAM Journal on Numerical Analysis, 42(2), pp.826-842.

[2] Bertrand, F. and Boffi, D., 2020. First order least-squares formulations for eigenvalue problems. IMA Journal of Applied Mathematics, to appear, arXiv preprint arXiv:2002.08145.

[3] Bertrand, F. and Boffi, D., 2020. Least-squares for linear elasticity eigenvalue problem. Comput. Math. Appl., to appear, arXiv preprint arXiv:2003.00449.

[4] Boffi, D., 2010. Finite element approximation of eigenvalue problems. Acta Numer., 19, pp.1-120. 
[5] Babuška, I. and Osborn, J., 1991. Eigenvalue problems.

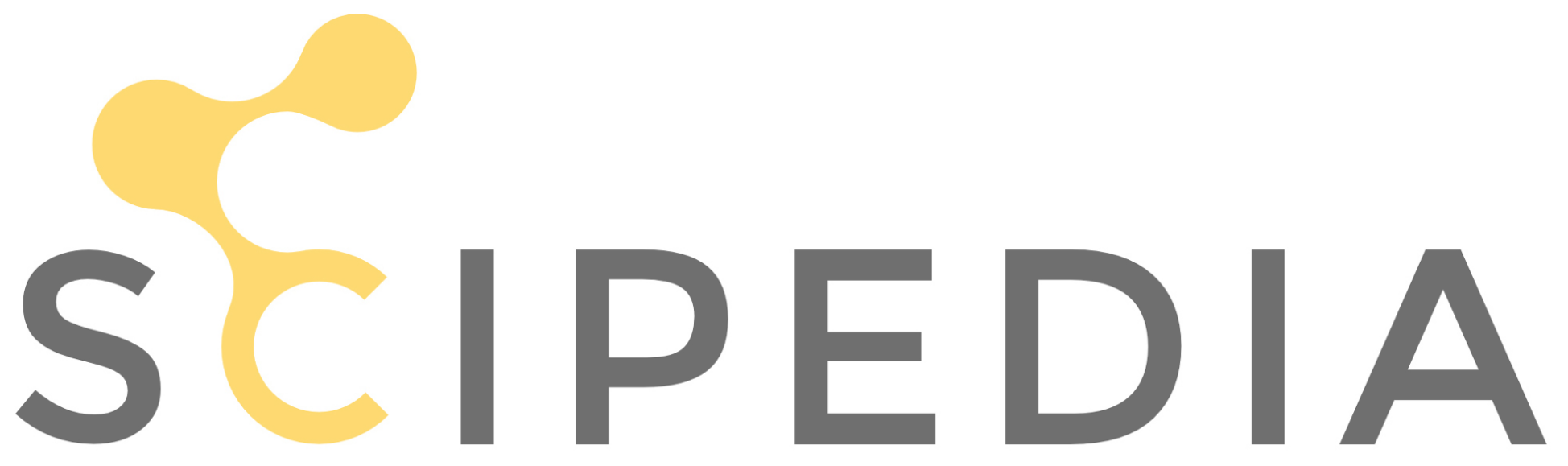

Register for free at https//www.scipedia.com to download the version without the watermark 